\title{
SIMPLE ANALYSIS OF FRAMED-TUBE STRUCTURES WITH MULTIPLE INTERNAL TUBES
}

\section{By Kang-Kun Lee ${ }^{1}$, Yew-Chaye Loo ${ }^{2}$, and Hong Guan ${ }^{3}$}

\begin{abstract}
Framed-tube system with multiple internal tubes is analysed using an orthotropic box beam analogy approach in which each tube is individually modelled by a box beam that accounts for the flexural and shear deformations, as well as the shear-lag effects. The method idealises the tubes-in-tube structure as a system of equivalent multiple tubes, each composed of four equivalent orthotropic plate panels capable of carrying axial loads and shear forces. By simplifying the assumptions in relation to the patterns of strain distributions in external and internal tubes, the structural analysis is reduced to the mere solution of a single secondorder linear differential equation. The proposed method, which is intended to be used as a tool for preliminary design purposes, can be applied for the analysis of framed-tube structures with single and multiple internal tubes, as well as those without internal tubes. The simplicity and accuracy of the proposed method is demonstrated through the analysis of three framed-tube structures (of different heights) without internal tubes. A 3-D frame analysis program and two existing approximate methods are also included in the comparative study. Furthermore, three other framed-tube structures with single, two and three internal tubes are analysed to verify the applicability and reliability of the proposed method.
\end{abstract}

\footnotetext{
${ }^{1}$ Research Assistant Professor, Advanced Research Structure Station, Hanyang University, Sungdong-Ku, Seoul 133-791, Korea

${ }^{2}$ Professor and Head, School of Engineering, Griffith University Gold Coast Campus, Queensland, Australia 9726

${ }^{3}$ Lecturer, School of Engineering, Griffith University Gold Coast Campus, Australia
} 


\section{INTRODUCTION}

Modern highrise buildings of the framed-tube system exhibit a considerable degree of shear-lag with consequential reduction in structural efficiency. Despite this drawback, framed-tube structures are widely accepted as an economical system for highrise buildings over a wide range of building heights. This is because in the framed-tube system the lateral load resisting elements are placed on the outer perimeter. The "tube" comprises closely spaced columns that are connected at each floor level by deep spandrel beams. Such buildings are usually equipped with service cores, which may house the lifts, emergency stairways, electrical and mechanical zones and other services. These cores referred to as the internal tubes are often designed to provide added lateral stiffness to the building; they also interact with each other as well as with the external tube. Framed-tube structures with multiple internal tubes, or tubes-in-tube structures, are widely used due to their high stiffness in resisting lateral loads and the availability of the internal tubes in supporting the vertical loads. The use of multiple internal tubes reduces the effect of shear-lag in the tubes and offers additional lateral stiffness to the overall structure.

The tube-tube interaction coupled with the existence of negative shear-lag in the tubes complicates the estimation of the structural performance and the accurate analysis of tubes in framed-tube system. Existing models for approximate analysis not only ignore the contribution of the internal tubes to the overall lateral stiffness but also neglect the negative shear-lag effects in the tubes. Thus, these models cater only for the structural analysis of the external tube but fail to consider the shear-lag

phenomenon of the internal tubes. As a result, they are inadequate in capturing the true behaviour of such structures. 
A number of approximate analysis methods (Coull, et al. 1975, 1978; Kwan 1994) were introduced for the analysis of single framed-tube structures (i.e. without internal tubes) by modeling the entire structure as an assemblage of equivalent orthotropic plate panels. Khan and Smith (1976) also developed an orthotropic plate analogy for the simplified analysis of such single-tube structures. However, these methods do not take into account the existence of negative shear-lag in the tube, which has an adverse effect on the structural behaviour. Chang (1985), on the other hand, proposed an approximate method that takes into account the overall lateral stiffness provided by the internal tube. However, only the analysis of lowrise structures was included in his study. In addition, the existence of the tube-tube interaction and the negative shear-lag, as well as the analysis of the internal tubes were not sufficiently discussed.

The occurrence of shear-lag has long been recognised in hollow box girders as well as in tubular structures. Foutch and Chang (1982) and Chang and Zheng (1987) observed the negative shear-lag phenomenon in box girders. Since then negative shear-lag effects have been considered in box girder design. However little effort has been made to understand the cause and the characteristics of such phenomenon. Recently, Kristek and Bauer (1993) and Singh and Nagpal (1995) also observed the existence of negative shear-lag in framed-tube structures. Yet, there is no comprehensive study on the net shear-lag behaviour or on the tube-tube interaction.

A typical framed-tube structure under lateral loading is shown in Fig. 1. The structure behaves differently from that predicted by the primary bending theory, in that the stress distribution in the flange wall panels is not uniform, and that in the web wall panels is nonlinear. These are illustrated in Fig. 2. This (nonlinear) phenomenon is referred to as "shear-lag". Positive shear-lag refers to the case 
where the stresses in the corner columns of the flange frame panels exceed those in the centre columns. This leads to the warping of the floor slabs which, in turn, causes the deformation of the interior partitions and other secondary components. In the case of negative shear-lag, where the stresses in the centre columns exceed those in the corner columns, local buckling on the compression side and cracking on the tension side of the flange frame may occur. In addition, the tube-tube interactive stresses, referred to as the additional bending stresses, would further complicate the shear-lag prediction.

The shear-lag phenomenon is more prominent in framed-tube structures with multiple internal tubes. In the present study, an analytical method is proposed for the accurate prediction of the axial stress distribution in the columns of each tube and the deflection of the structure under lateral loading. The proposed method takes into account the net shear-lag effects and the additional bending stresses in the tubes. The added lateral stiffness provided by the internal tubes is also considered. The numerical analysis so developed is based on the minimum potential energy principle in conjunction with the variational approach.

The simplicity and accuracy of the proposed method is demonstrated through the analysis of three single tube structures with 30,50 and 70 storeys. The results due to the proposed method are compared with those produced by a 3-D frame analysis program (ETABS 1989). The analytical results from two existing approximate methods due to Coull, et al. $(1975,1978)$ and Kwan $(1994)$ are also included in the comparative study.

To verify the applicability and reliability of the proposed method, three other 40-storey framed-tube structures with single, two and three internal tubes are also 
analysed. The results for lateral deflections and the axial stresses in the corner and centre columns of the flange frame panels in each of the tubes are compared with those given by the 3-D frame analysis program. The shear-lag phenomenon is also discussed; so are the additional bending stresses due to the tube-tube interaction.

\section{FRAMED-TUBE STRUCTURES WITH MULTIPLE INTERNAL TUBES}

A discrete tubes-in-tube structure may be modelled as an assemblage of equivalent orthotropic plate panels. Consequently, a framed-tube structure may be analysed as a continuum. Fig. 3 shows a typical framed-tube structure with multiple internal tubes (two in this case). Each of the tubes is composed of four equivalent orthotropic plate panels. All framed-tubes under consideration consist of an assemblage of such plate panels of uniform thickness in vertical planes.

The following assumptions are made to simplify the modelling process and the associated analysis:

(a) The floor slabs in the structure are considered to be rigid diaphragms within their own plane. Thus, the relative lateral displacements between the tubes are negligible at each level.

(b) The behaviour of the structure is linear and elastic.

(c) No local bending exists in the joint areas or in the panels.

(d) The spacings of the beams and columns are uniform throughout the building height.

(e) Both beams and columns are of uniform cross section throughout the building height.

(f) The axial stiffnesses of the beams and columns may be represented, respectively, by the elastic moduli in the horizontal ( $x$ or $y$ ) and vertical ( $z$ ) 
directions at each frame panel (see Fig. 3). The shear stiffness is represented by the shear modulus.

\section{SHAPE FUNCTIONS}

Fig. 3 indicates that the framed-tube structure has two horizontal axes of symmetry ( $x$ and $y$ ) with the vertical axis ( $z$ ) passing through the centre of the cross section of the structure. Based on this assumption, the strain distributions in the external web frame panels 1 and 2 are identical in the 2 tubes-in-tube structure shown in Fig. 4. Note that the strain distributions in the external flange frame panels (3 and 4) are equal but opposite. Since the internal web frame panels 5, 6, 7 and 8 are identical in size, their deflection profiles are also the same. The strain distributions in all these four internal web frame panels are therefore identical. It is further assumed that the strain distributions in the flange frame panels of internal tubes are symmetrical about the central vertical axis (z). As a result, the strain distributions in such panels are identical in magnitude. Similar assumptions are also applied to the framed-tube structures with different numbers of internal tubes.

The analytical method proposed herein has the following characteristics:

(a) Reissner's function (Reissner 1945) is modified to account for the independent distribution of the vertical displacement in the flange frame panels, thereby taking into consideration the net shear-lag.

(b) The effect of shear-lag in the external and internal tubes is considered in assessing the global behaviour of the tubes-in-tube structures.

(c) The overall lateral stiffness provided by the internal tubes is considered.

(d) The additional bending stresses, due to the tube-tube interaction, are evaluated to assess the effect of the net shear-lag. 
(e) The proposed method is applicable for the analysis of framed-tube structures with and without internal tubes.

\section{Vertical Displacements in External Tube}

To estimate the positive and negative shear-lags in the orthotropic plate panels, the distribution of vertical displacement in the flange frame panel is considered to closely follow the Reissner's function (Reissner 1945). However, certain modification is necessary to replace the parabolic variation by a cubic one. A cubic function is chosen for its greater flexibility and versatility with negligible increase in computational effort. The vertical displacement distribution in the web frame panel is also assumed to be cubic. Figs. 5(a) and 5(b) show, respectively, the general shapes for the displacement distributions in the flange and web frame panels. The general expressions for the displacement distributions are assumed as

$$
U_{1}(z, y)=c\left[\frac{d w}{d z}+\left(1-\left(\frac{y}{b}\right)^{3}\right) u_{1}(z)\right] \quad \text { for flange frame panel }
$$

and $\quad U_{2}(z, x)=\left[\frac{d w}{d z} x+\left(\frac{x}{c}-\left(\frac{x}{c}\right)^{3}\right) u_{2}(z)\right] \quad$ for web frame panel

where $u_{1}(z)$ and $u_{2}(z)$ are the undetermined displacement functions including shearlag coefficients due to the shear deformation; $w$ is the deflection of the structure; $b$ and $c$ are the half-widths of the flange and web frame panels of the external tube, respectively, and, $x, y$ and $z$ are the coordinates of the three rectangular axes. 


\section{Vertical Displacements in Internal Tube}

The general expressions, Eqs. (1a) and (1b), for the displacement distributions in the external tube can also be applied to those in the flange and web frame panels of the multiple internal tubes. Fig. 6 shows a typical plan of equivalent tube structure with multiple internal tubes. When the number of internal tubes, $N$, is an even number ( $N=2,4,6$ or 8 , etc), the displacement distribution in the flange frame, $U_{i 1}(z, y)$, can be expressed as

$$
U_{i 1}(z, y)=c_{i}\left[\frac{d w}{d z}+\left(1+8\left(\frac{y-n_{1}}{2 b_{i}}-\frac{1}{2}\right)^{3}\right) u_{i 1}(z)\right]
$$

for $n_{1} \leq y \leq n_{2}$; and, for $n_{2} \leq y \leq n_{3}$,

$$
U_{i 1}(z, y)=c_{i}\left[\frac{d w}{d z}+\left(1-8\left(\frac{y-n_{1}}{2 b_{i}}-\frac{1}{2}\right)^{3}\right) u_{i 1}(z)\right]
$$

where $n_{1}=(n-1) a / 2+(n-2) b_{i}, n_{2}=(n-1) a / 2+(n-1) b_{i}, n_{3}=(n-1) a / 2+n b_{i}$ and $n=2(N / 2)$ ! ; in which $b_{i}$ and $c_{i}$ are the half-widths of the flange and web frame panels of the internal tubes, respectively. In Eqs. (2a) and (2b), $u_{i 1}(z)$ is the undetermined displacement function including shear-lag coefficients due to the shear deformation. For a framed-tube structure with an odd number of internal tubes, a similar consideration follows. The displacement distribution in the flange frame of the internal tubes can be expressed as 


$$
U_{i 1}(z, y)=c_{i}\left[\frac{d w}{d z}+\left(1-\left(\frac{y}{b_{i}}\right)^{3}\right) u_{i 1}(z)\right] \quad \text { for } 0 \leq y \leq b_{i}
$$

For $n_{1} \leq y \leq n_{2}$ and $n_{2} \leq y \leq n_{3}$, respectively, the displacement distribution has the identical expression as Eqs. (2a) and (2b). However, with the same expressions of $n_{1}, n_{2}$ and $n_{3}, n=[(N-1) / 2] ! \times 2+1$ and $N=3,5$, or 7 , etc.

An assumption analogous to that for the displacement distribution in the external web frame panel is also applied to the internal web frame panels (see Fig. 6). Hence the displacement distribution, $U_{i 2}(z, x)$, can be expressed as

$$
U_{i 2}(z, x)=\left[\frac{d w}{d z} x+\left(\frac{x}{c_{i}}-\left(\frac{x}{c_{i}}\right)^{3}\right) u_{i 2}(z)\right]
$$

where $u_{i 2}(z)$ is the undetermined displacement function including shear-lag coefficients due to the shear deformation. Differentiating Eqs. (1), (2), (3) and (4) with respect to $z$ yields the vertical strains in the flange and web frame panels of the external and internal tubes.

\section{SOLUTION METHOD}

The equilibrium equation of the overall moment at any building height is 


$$
\begin{gathered}
M(z)=2 \int_{-b}^{b} E \varepsilon_{z f} t c d y+2 \int_{-c}^{c} E \varepsilon_{z w} t x d x+4 A_{C} c^{2} E \varepsilon_{c}+ \\
\sum_{m=1}^{N}\left[2 m \int_{-b_{i}}^{b_{i}} E \varepsilon_{z i f} t_{i} c_{i} d y+2 m \int_{-c_{i}}^{c_{i}} E \varepsilon_{z i w} t_{i} x d x+4 m A_{c_{i}} c_{i}{ }^{2} E \varepsilon_{c_{i}}\right]
\end{gathered}
$$

where $E$ is the modulus of elasticity; $t$ and $t_{i}$ are respectively the equivalent orthotropic panel thicknesses in the external and internal tubes; $A_{c}$ and $A_{c i}$ are the cross-sectional areas of the corner columns in the external and internal tubes, respectively; $\varepsilon_{C}$ and $\varepsilon_{C_{i}}$ are respectively the corner column strains in the external and internal tubes; $\varepsilon_{z f}$ and $\varepsilon_{z w}$ are the strains in the external flange and web frame panels, respectively; $\varepsilon_{\text {zif }}$ and $\varepsilon_{\text {ziw }}$ are the strains in the internal flange and web frame panels, respectively.

Substituting Eqs. (1), (2), (3) and (4) into Eq. (5) and integrating with respect to $z$ yields

$$
\begin{aligned}
& u_{2}^{\prime}(z)=-\frac{15 b}{2} u_{1}^{\prime}(z) \quad \text { for the external tube } \\
& u_{i 2}^{\prime}(z)=-\frac{15 b_{i}}{2} u_{i 1}^{\prime}(z) \quad \text { for single internal tube } \\
& u_{i 2}^{\prime}(z)=-\frac{45 b_{i}}{8} u_{i 1}^{\prime}(z) \quad \text { for more than two internal tubes }
\end{aligned}
$$

and 
As a result of Eq. (6), the vertical strain distributions can be simply expressed in terms of the two undetermined strain functions, $u_{1}^{\prime}(z)$ and $u_{i 1}^{\prime}(z)$.

The numerical analysis of the stress distributions is carried out using the principle of minimum potential energy in conjunction with the variational approach (Ketter, Lee, and Prawel 1979). The total potential energy (V) of a framed-tube structure with multiple internal tubes is obtained by summing up the total strain energy of the external and internal tubes $\left(V_{s}\right.$ and $\left.V_{i s}\right)$ and the potential energy of the loading system as applied to the external and internal tubes $\left(V_{L}\right.$ and $\left.V_{i L}\right)$. Or,

$$
V=V_{s}-V_{L}+V_{i s}-V_{i L}
$$

where $\left(V_{S}-V_{L}\right)$ and $\left(V_{i s}-V_{i L}\right)$ are, respectively, the total potential energies of the external and internal tubes. Note that $V_{s}, V_{i s}, V_{L}$ and $V_{i L}$ are functions of $z$ and their expressions may be found elsewhere (Lee, 1999). The total potential energy given in Eq. (7) may be rewritten symbolically in a functional form with respect to $z, w^{\prime \prime}, u_{1}, u_{1}^{\prime}, u_{i 1}$ and $u_{i 1}^{\prime}$ as

$$
V=\int_{0}^{H} F\left(z, w^{\prime \prime}, u_{1}, u_{1}^{\prime}, u_{i 1}, u_{i 1}^{\prime}\right) d z
$$

The total potential energy of the entire system must be minimised in conjunction with the variational approach. When a set of boundary conditions is applied, the governing differential equations can then be derived. Considering that the structure is fixed at its base $(z=H)$, the geometric boundary conditions are 


$$
w(H)=u_{1}(H)=u_{i 1}(H)=0 \quad \text { at } z=H ;
$$

and

$$
u_{1}^{\prime}(0)=u_{i 1}^{\prime}(0)=0 \quad \text { at } z=0
$$

where $H$ is the structural height. Subject to the boundary conditions given in Eq. (9), the governing differential equations can be expressed as

$$
u_{1}^{\prime \prime}-u_{1} X^{2}=P_{e}(z) Y
$$

and

$$
u_{i 1}^{\prime \prime}-u_{i 1} X_{1}^{2}=P_{i}(z) Y_{1}
$$

where $P_{e}(\mathrm{z})$ and $P_{i}(\mathrm{z})$ are the total shear forces in the external and internal tubes, respectively. In Eq. (10), $X, Y, X_{1}$ and $Y_{1}$ are the constants to be determined according to the structural properties and the geometric conditions (Lee, 1999).

The general solutions for the two non-homogeneous equations in Eq. (10) are

$$
u_{1}(z)=A \sinh X z+B \cosh X z+C P_{e}(z) Y
$$

and

$$
u_{i 1}(z)=A \sinh X_{1} z+B \cosh X_{1} z+C P_{i}(z) Y_{1}
$$

where $A, B, C, D, E$ and $F$ are the constants to be determined according to the loading conditions. 


\section{DEFLECTION AND STRESSES}

\section{Deflections}

The deflection of the structure, $w$, can be derived from the shear strain, $\gamma_{x z}$, for the web frame. Or,

$$
\frac{\partial w}{\partial z}=\gamma_{x z}-\frac{\partial U_{2}(z, x)}{\partial x}=\frac{\tau_{x z}}{G}-\frac{\partial U_{2}(z, x)}{\partial x}
$$

where $\gamma_{x z}=\frac{\partial U_{2}(z, x)}{\partial x}+\frac{\partial w}{\partial z}, \tau_{x z}$ is the shear stress in the web frame panel of the external tube and $G$ is the shear modulus of the equivalent tube. The expressions of $\tau_{x z}$ and $\frac{\partial U_{2}(z, x)}{\partial x}$ are available elsewhere (Lee, 1999). Integrating Eq. (12) with respect to $z$ and considering the boundary conditions given in Eq. (9) yield the horizontal deflection of the structure subjected to a lateral load.

\section{Stress Distributions}

The deflection of the structure can be derived as

$$
w^{\prime \prime}=-\frac{1}{E I}\left(M-\left(u_{1}^{\prime} \alpha_{1}+u_{i 1}^{\prime} \beta_{1}\right)\right)=-\frac{1}{E I}\left(M+M_{S}\right)
$$

where $M_{s}=-\left(u_{1}^{\prime} \alpha_{1}+u_{i 1}^{\prime} \beta_{1}\right)$ is an additional moment induced by the shear deformation due to the tube-tube interaction. $I$ is the second moment of area of the 
tubes-in-tube system. Substituting Eq. (13) into the vertical strains of the external and internal tubes and multiplying by $E$ yield the bending stresses in the external tube. Or,

$$
\sigma_{z f}=-E\left[\frac{M(z)}{E I} c-\left(1-\frac{y^{3}}{b^{3}}+\frac{I_{N}}{I}\right) c u_{1}^{\prime}(z)-\frac{I_{i N}}{I} c u_{i 1}^{\prime}(z)\right]=E w^{\prime \prime} c+\sigma_{f s}
$$

for the external flange frame panel; and

$$
\sigma_{z w}=-E\left[\frac{M(z)}{E I} x-\left(\frac{15}{2} \frac{b}{c} x-\frac{15 b}{2}\left(\frac{x}{c}\right)^{3}-\frac{I_{N}}{I} x\right) u_{1}^{\prime}(z)-\frac{I_{i N}}{I} x u_{i 1}^{\prime}(z)\right]=E w^{\prime \prime} x+\sigma_{w s}
$$

for the external web frame panel. In Eq. (14), $\sigma_{f s}$ and $\sigma_{w s}$ are, respectively, the additional bending stresses in the flange and web frame panels due to the tube-tube interaction. $I_{N}$ and $I_{i N}$ are the second moments of area of the flange panels in the external and internal tubes, respectively;

The same procedure can be applied to evaluate the bending stresses in the flange and web frame panels of the internal tubes. This gives, for the internal flange frame panels,

$$
\sigma_{z i f}=-E\left[\frac{M_{i}(z)}{E I_{i}} c_{i}-\left(1-\frac{y^{3}}{b_{i}^{3}}+\frac{I_{i N}}{I_{i}}\right) c_{i} u_{i 1}^{\prime}(z)\right]=E w^{\prime \prime} c_{i}+\sigma_{f i s}
$$

for a structure with single internal tube; and 
$\sigma_{\text {zif }}=-E\left[\frac{M_{i}(z)}{E I_{i}} c_{i}-\left(1 \pm 8\left(\frac{y-n_{1}}{2 b_{i}}-\frac{1}{2}\right)^{3}+\frac{I_{i N}}{I_{i}}\right) c_{i} u_{i 1}^{\prime}(z)\right]=E w^{\prime \prime} c_{i}+\sigma_{f i s}$

for a structure with more than two internal tubes. In Eq. (15), $I_{i}$ is the second moment of area of the internal tube. Note that the " \pm " sign in Eq. (15b) indicates that "+" is used when $n_{1} \leq y \leq n_{2}$ and "-" is used when $n_{2} \leq y \leq n_{3}$. Note also that $n_{1}, n_{2}$ and $n_{3}$ take different values for even and odd numbers of internal tubes.

The bending stress in the internal web frame panels is

$\sigma_{\text {ziw }}=-E\left[\frac{M_{i}(z)}{E I_{i}} x-\left(\frac{15}{2} \frac{b_{i}}{c_{i}} x-\frac{15 b_{i}}{2}\left(\frac{x}{c}\right)^{3}-\frac{I_{i N}}{I_{i}} x\right) u_{i 1}^{\prime}(z)\right]=E w^{\prime \prime} x+\sigma_{w i s}$

In Eqs. (15) and (16), $\sigma_{\text {fis }}$ and $\sigma_{\text {wis }}$ are, respectively, the additional bending stresses in the internal flange and web frame panels due to the tube-tube interaction; $M(z)$ and $M_{i}(z)$ are the total bending moment of the entire system and that of the internal tubes, respectively. Note that the behaviour of the tubes-in-tube structure, when subjected to the lateral load, is similar to that of a frame-shear wall structure (Schueller 1992). The moment of the internal tubes, $M_{i}(z)$, can therefore be derived from the analysis of the frame-shear wall structure (Smith and Coull 1991). 


\section{NUMERICAL APPLICATIONS}

\section{Example 1 - Analysis of Framed-tube Structures without Internal Tubes}

To demonstrate the simplicity and accuracy of the proposed method, three single framed-tube structures of 30,50 and 70 storeys are analysed. The results due to the proposed method are compared with those produced by a 3-D frame analysis program (ETABS 1989). Also included in the comparative study are the analysis results from the two existing approximate methods due to Coull, et al. (1978) and Kwan (1994). The capabilities of the proposed method and those of the two approximate methods are compared in Table 1.

The plan and sectional views of the three structures are shown in Fig. 7. Each of the three structures is $30 \mathrm{~m} \times 30 \mathrm{~m}$ in plan and has a $3.0 \mathrm{~m}$ storey height. The centre-to-centre column spacing is $2.5 \mathrm{~m}$. A uniformly distributed lateral load of $88.24 \mathrm{kN} / \mathrm{m}$ is applied along the entire height of the structure. The cross-sectional area of all the columns and beams is taken to be $0.64 \mathrm{~m}^{2}$, and the Young's modulus $E$ and the shear modulus $G$ are $2.06 \times 10^{10} \mathrm{~N} / \mathrm{m}^{2}$ and $0.2 \times 10^{10} \mathrm{~N} / \mathrm{m}^{2}$, respectively.

Figs. 8 and 9 illustrate, respectively, the lateral deflections of the three framedtube structures and the corresponding axial stresses in the centre and corner columns of the flange frame panels. Compared to the 3-D frame analysis results, the proposed method yields good correlations in both deflection and axial stresses for all three structures. On the other hand, the two existing approximate methods give rather poor results in deflection, especially at higher storeys. Some discrepancies are also found between the existing methods and the 3-D frame analysis in predicting the column axial stresses. This is particularly true for Kwan's method (1994) in predicting the 
centre-column axial stress in the flange frame panel. It may be further observed from Fig. 8 that the proposed analysis is more accurate for structures with over 30 storeys. In addition, it is found from the comparisons that the proposed method requires minimal data preparation effort, and for each analysis, the personal computer running time is absolutely negligible when compared with the 3-D frame analysis.

In view of its simplicity, efficiency and accuracy, the proposed method is considered to be a suitable design tool for framed-tube structures, particularly at the preliminary stages where numerous analysis iterations need to be carried out.

\section{Example 2 - Analysis of Tube(s)-in-Tube Structures}

To further demonstrate the applicability and reliability of the proposed method, three 40-storey framed-tube structures with single, two and three internal tubes are analysed and the results are compared with the 3-D frame analysis program (ETABS 1989). The structural dimensions are the same as those adopted in Example 1. Each structure is subjected to a $88.24 \mathrm{kN} / \mathrm{m}$ lateral load. The second moment of area of each internal tube is taken to be $90 \mathrm{~m}^{2}$. The plan views of the three structures are shown in Fig. 10. The geometry of the structures are summarised in Table 2.

The deflections of the three tube(s)-in-tube structures and the axial stress distributions, along $\mathrm{z} / \mathrm{H}$, in the centre and corner columns of the flange frame panels are obtained using the proposed method and the 3-D frame analysis program. They are presented in Figs. 11 and 12, respectively. It can be seen in Fig. 11 that the discrepancy between the deflection curves is more pronounced in the lower storeys. This phenomenon is due to the effects of the additional lateral stiffness provided by the internal tubes, which is not considered in the 3-D frame analysis 
program. The column axial stress distributions in the flange frame panels shown in Fig. 12 indicate that the stress reversals for the internal tubes take place at two level points (near the middle level and top level); for the external tube, the stress reversal takes place at near-top level only. It is further observed that all three structures having the same second moment of area of the internal tubes, show similar characteristics in deflection and axial stresses. This implies that increasing the number of internal tubes has little effect on the structural behaviour of the framedtube structures. However, when the second moment of area of the internal tubes increases, the structural behaviour would vary significantly between structures with different numbers of internal tubes.

Shown in Fig. 13 are the distributions of the additional bending stresses in the centre and corner columns of the flange frame panels for the three structures. It is found that as the second moment of area of each internal tube is identical, increasing the number of internal tubes gradually reduces the increment in the additional bending stresses from centre to corner columns in the internal tubes. In other words, a reduction occurs in bending stresses between centre and corner columns. As a result, the shear-lag is also reduced. However, the number of internal tubes does not have much effect on the additional bending stresses in the external tube. It is further observed that the effect of the positive shear-lag is greater at the bottom of the structures, whereas the negative shear-lag in the external tube occurs at around $1 / 4$ of the building height. For all three structures, the shear-lag reversal point for the internal tubes locates at a lower level than that for the external tube. 


\section{CONCLUSION}

Based on the minimum potential energy principle in conjunction with the variational approach, a simple mathematical model is proposed for the approximate analysis of framed-tube structures with multiple internal tubes. The structures are modelled as a box assemblage of the orthotropic plate panels, each composed of horizontal beams and vertical columns. In the proposed method, Reissner's function is modified to account for the independent distribution of the vertical displacement in the flange frame panels of each tube, thereby taking the net shear-lag into consideration. By simplifying the assumptions in relation to the patterns of the vertical displacement distributions in the tubes, the complex three-dimensional structural analysis is reduced to the mere solution of a single second-order linear differential equation. The proposed method is applicable to the analysis of framedtube structures with any number of internal tubes. This is an advancement over the existing methods which are restricted to the single tube structures. Note that the structures to be analysed should preferably possess a high degree of regularity for the simplification of the analysis procedure. The bay widths and storey heights should be the same over the major part of the structures, and the same member sizes should be used in both the horizontal and vertical directions.

The accuracy, simplicity and reliability of the proposed method are verified through the comparisons with the two existing simplified methods and a 3-D frame analysis program. The comparative study is carried out based on the analysis of various types of framed-tube and tube(s)-in-tube structures. The accuracy and economy of the proposed method is confirmed. 
The additional lateral stiffness due to the tube-tube interaction is also accounted for in the analysis. The additional bending stresses are observed to have significant effect on the shear-lag phenomenon. In comparison with the 3-D frame analysis program, the only other approach available for the tubes-in-tube system, the proposed method provides similarly accurate results in predicting the deflection response and the column axial stress distributions.

The proposed method is simple, accurate, economical and reliable. It is especially suitable for use at preliminary design stages where a large number of structures with different features are required to be analysed repeatedly. 


\section{APPENDIX I. REFERENCES}

Chang, P. C. (1985). “Analytical modelling of tube-in-tube structure.” J. Struct. Engrg., ASCE, 111(6), 1326-1337.

Coull, A., and Ahmed, A. A. (1978). "Deflections of framed-tube structures." $J$. Struct. Engrg., ASCE, 104(5), 857-862.

Coull, A., and Bose, B. (1975). "Simplified analysis of framed-tube structures." $J$. Struct. Engrg., ASCE, 101(11), 2223-2240.

Chang, S. T., and Zheng, F. Z. (1987). "Negative shear lag in cantilever box girder with constant depth.” J. Struct. Engrg., ASCE, 113(1), 20-33.

ETABS(1989). Three Dimensional Analysis of Building System. Computers and Structures Inc., Berkerly, California, U.S.A.

Foutch, D. A., and Chang, P. C. (1982). “A shear lag anomaly.” J. Struct. Engrg., ASCE, 108(ST7), 1653-1657.

Ketter, R. L., Lee, G. C., and Prawel, S. P. (1979). Structural Analysis and Design. MaGraw-Hill, Inc., New York.

Khan, A. H., and Smith, B. S. (1976). "A simple method of analysis for deflection and stresses in wall-frame structures.” Building and Environment, 11, 69-78.

Kristek, V., and Bauer, K. (1993). "Stress distribution in front columns of high rise buildings.” J. Struct. Engrg., ASCE, 119(5), 1464-1483.

Kwan, A. K. H. (1994). "Simple method for approximate analysis of framed tube structures.” J. Struct. Engrg., ASCE, 120(4), 1221-1239.

Lee, K.K. (1999). Orthotropic Box Beam Analogy for Analysis of Framed Tube Structures with Multiple Internal Tubes, PhD Thesis, Griffith University, Australia.

Reissner, E. (1945). "Analysis of shear lag in box beam by the principle of minimum potential energy.” Quarterly of Applied Mathematics, 4(3), 268-278. 
Schueller, W. (1992). Highrise Building Structures. Robert E. Krieger Publishing Co., New York.

Singh, Y., and Nagpal, A. K. (1995). "Negative shear lag in framed-tube buildings.” J. Struct. Engrg., ASCE, 120(11), 3105-3121.

Smith, B. S., and Coull, A. (1991). Tall Building Structures: Analysis and Design. A Wiley-Interscience Publication, John Wiley \& Sons, Inc., Toronto, Canada. 


\section{APPENDIX II. NOTATION}

The following symbols are used in this paper:

$A_{c}, A_{c i}=$ cross-sectional areas of corner columns in the external and internal tubes, respectively

$b, c=$ half-widths of the external flange and web frame panels, respectively

$b_{i}, c_{i}=$ half-widths of the internal flange and web frame panels, respectively

$E=$ modulus of elasticity

$G=$ shear modulus of elasticity

$H=$ structural height

$I_{i}, I=$ second moments of area of the internal tube and the entire tubes-in-tube system, respectively

$I_{N}, I_{i N}=$ second moments of area of the flange panel in the external and internal tubes, respectively

$M_{S}=$ additional moment

$M(z), M_{i}(z)=$ total bending moment of the entire system and bending moment of the internal tube

$N=$ number of internal tubes

$P_{e}(\mathrm{z}), P_{i}(\mathrm{z})=$ shear forces of the external and internal tubes, respectively 


$$
\begin{aligned}
t, t_{i}= & \text { equivalent orthotropic panel thicknesses of the external } \\
& \text { and internal tubes, respectively }
\end{aligned}
$$

$U_{1}(z, y), U_{2}(z, x)=$ displacement distributions in the external flange and web frame panels, respectively

$U_{i 1}(z, y), U_{i 2}(z, x)=$ displacement distributions in the internal flange and web frame panels, respectively

$u_{1}(z), u_{2}(z)=$ undetermined functions for displacement distribution in the external flange and web frame panels, respectively

$u_{i 1}(z), u_{i 2}(z)=$ undetermined functions for displacement distribution in the internal flange and web frame panels, respectively

$u_{1}^{\prime}(z), u_{2}^{\prime}(z)=$ undetermined functions for strain distribution in the external flange and web frame panels, respectively

$u_{i 1}^{\prime}(z), u^{\prime}{ }_{i 2}(z)=$ undetermined functions for strain distribution in the internal flange and web frame panels, respectively

$$
V=\text { total potential energy }
$$

$V_{i s}(z), V_{i L}(z)=$ strain and potential energies of the load system in the internal tubes, respectively

$V_{S}(z), V_{L}(z)=$ strain and potential energies of the load system in the external tube, respectively

$$
\begin{aligned}
w(z) & =\text { deflection } \\
X, Y & =\text { variables for undetermined function, } u_{1}(z) \\
X_{1}, Y_{1} & =\text { variables for undetermined function, } u_{i 1}(z)
\end{aligned}
$$




$$
\begin{aligned}
x, y, z= & \text { coordinates of three rectangular axes } \\
\varepsilon_{C}, \varepsilon_{c i}= & \text { corner column strains in the external and internal tubes, } \\
& \text { respectively } \\
\varepsilon_{z f}, \varepsilon_{z w}= & \text { strains in the external flange and web frame panels, } \\
& \text { respectively } \\
\varepsilon_{z i f}, \varepsilon_{z i w}= & \text { strains in the internal flange and web frame panels, } \\
& \text { respectively } \\
\gamma_{x z}= & \text { shear strains in the web frame panel of the external tube } \\
\sigma_{f s}, \sigma_{w s}= & \text { additional bending stresses in the external flange and web } \\
& \text { frame panels, respectively } \\
\tau_{x z}= & \text { shear stress in the web frame panel of the external tube } \\
\sigma_{f i s}, \sigma_{w i s}= & \text { additional bending stresses in the internal flange and web } \\
& \text { frame panels, respectively } \\
\sigma_{z i f}, \sigma_{z i w}= & \text { bending stresses in the flange and web frame panels of } \\
\sigma_{z f}, \sigma_{z w}= & \text { bending stresses in the flange and web frame panels of } \\
& \text { external tube, respectively }
\end{aligned}
$$




\section{List of figure captions}

Fig. 1 Typical Framed-Tube Structure

Fig. 2 Stress Distribution of Laterally Loaded Tubes-in-Tube Structure

Fig. 3 Equivalent Tube Structure with Multiple (Two) Internal Tubes

Fig. 4 Plan of Framed-Tube Structure with Two Internal Tubes

Fig. 5 Distributions of Vertical Displacements in Flange and Web Frame Panels

Fig. 6 Typical Plan of Equivalent Tubes-in-Tube Structure

Fig. 7 Geometry of Three Framed-Tube Structures

Fig. 8 Deflections of Three Framed-Tube Structures

Fig. 9 Axial Stresses in Columns of Flange Frame Panels for Three FramedTube Structures

Fig. 10 Plans of Three Framed-Tube Structures with Different Number of Internal Tubes

Fig. 11 Deflections of Framed Tube Structures with Multiple Internal Tubes

Fig. 12 Axial Stresses in Centre and Corner Columns of Flange Frame Panels for Framed-Tube Structures with Multiple Internal Tubes

Fig. 13 Distributions of Additional Bending Stresses in Centre and Corner Columns of Flange Frame Panels for Framed Tube Structures with Multiple Internal Tubes 
Table 1. Comparison of the proposed method and two approximate methods

\begin{tabular}{|c|c|c|c|}
\hline \hline $\begin{array}{c}\text { Characteristics } \\
\text { of the method } \\
(1)\end{array}$ & $\begin{array}{c}\text { Proposed } \\
\text { method }\end{array}$ & $\begin{array}{c}\text { Coull, et al. } \\
(1978)\end{array}$ & $\begin{array}{c}\text { Kwan } \\
(1994)\end{array}$ \\
\hline Methodology & \multicolumn{2}{|c|}{ Equivalent orthotropic panel } \\
\hline Applied structural & $\begin{array}{c}\text { Tubes-in-tube } \\
\text { Tube-in-tube } \\
\text { system }\end{array}$ & Tube & Tube \\
\hline $\begin{array}{c}\text { Consideration of } \\
\text { negative shear-lag }\end{array}$ & Yes & No & No \\
\hline Stress distribution \\
in web panel
\end{tabular}


Table 2. Geometry of three tube(s)-in-tube structures

\begin{tabular}{|c|c|c|c|}
\hline $\begin{array}{l}\text { Structural } \\
\text { data } \\
\text { (1) }\end{array}$ & $\begin{array}{l}\text { Tube- } \\
\text { in- } \\
\text { tube } \\
\text { (2) }\end{array}$ & $\begin{array}{l}\text { Tube structure } \\
\text { with two } \\
\text { internal tubes } \\
\text { (3) }\end{array}$ & $\begin{array}{c}\text { Tube structure } \\
\text { with three } \\
\text { internal tubes } \\
\text { (4) }\end{array}$ \\
\hline $\begin{array}{l}\text { Column and beam } \\
\text { sizes in external } \\
\text { tube }(\mathrm{cm} \times \mathrm{cm})\end{array}$ & \multicolumn{3}{|c|}{$80 \times 80$} \\
\hline $\begin{array}{l}\text { Column and beam } \\
\text { sizes in internal } \\
\text { tube }(\mathrm{cm} \times \mathrm{cm})\end{array}$ & $91 \times 91$ & $80 \times 80$ & $72 \times 72$ \\
\hline $\begin{array}{l}\text { Size of external } \\
\text { tube }(\mathrm{m} \times \mathrm{m})\end{array}$ & \multicolumn{3}{|c|}{$30 \times 15$} \\
\hline $\begin{array}{l}\text { Size of internal } \\
\text { tube }(\mathrm{m} \times \mathrm{m})\end{array}$ & $15 \times 5$ & 2@7.5×5 & $3 @ 5 \times 5$ \\
\hline $\begin{array}{l}\text { Total storey } \\
\text { height (m) }\end{array}$ & \multicolumn{3}{|c|}{120} \\
\hline
\end{tabular}




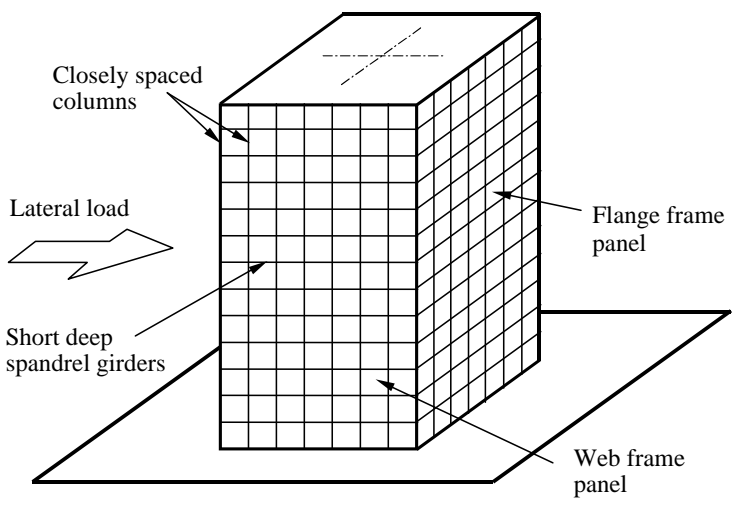

Fig. 1

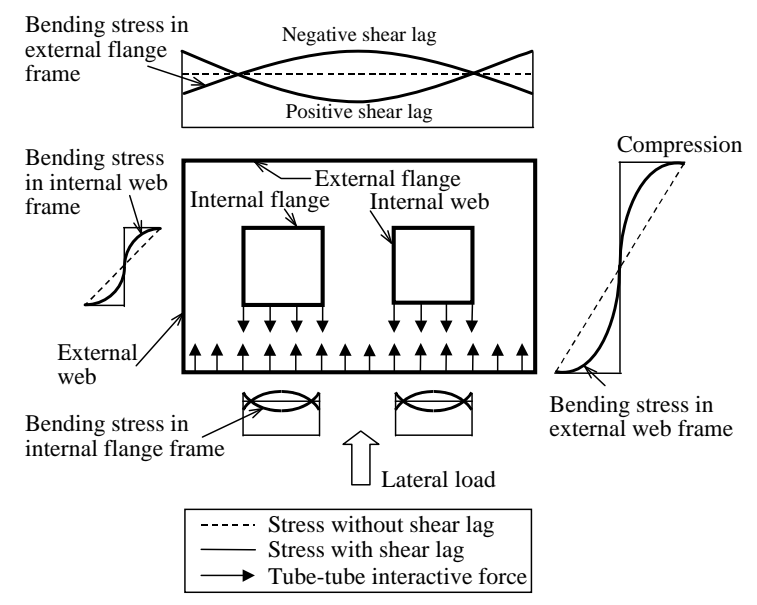

Fig. 2

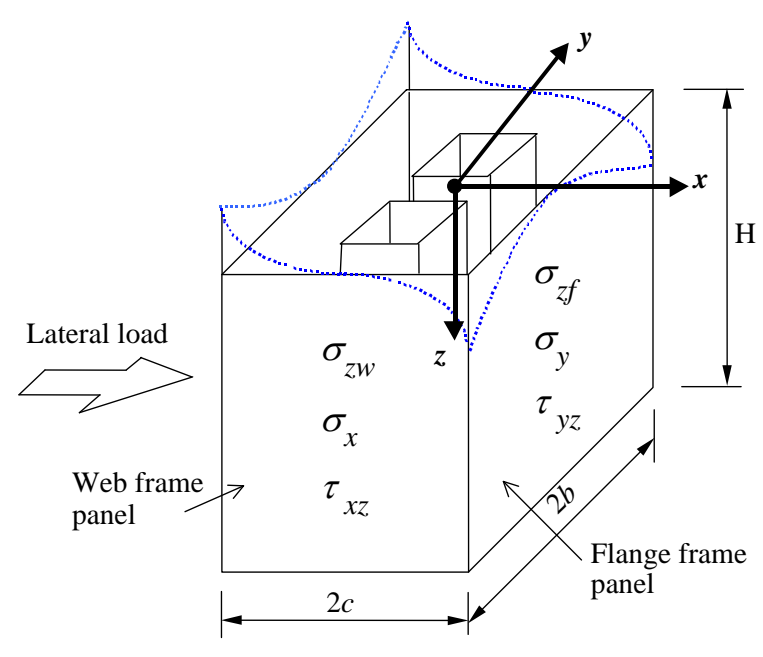

Fig. 3

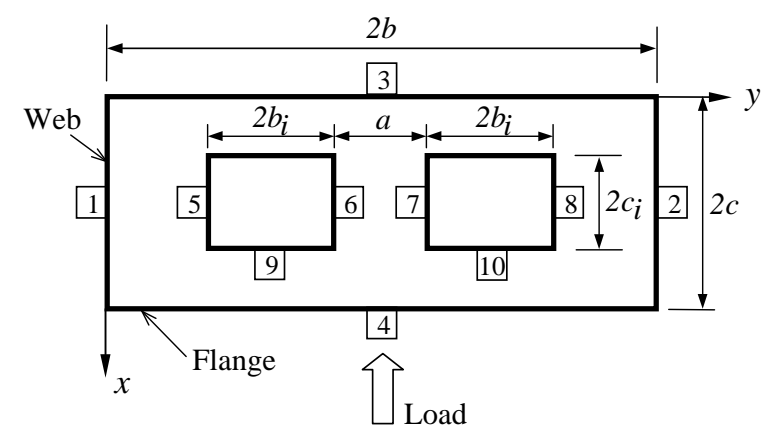

Fig. 4

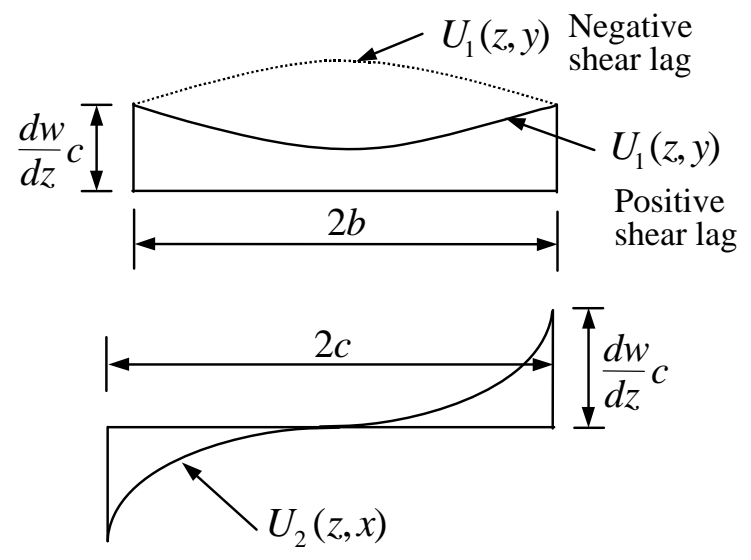

Fig. 5

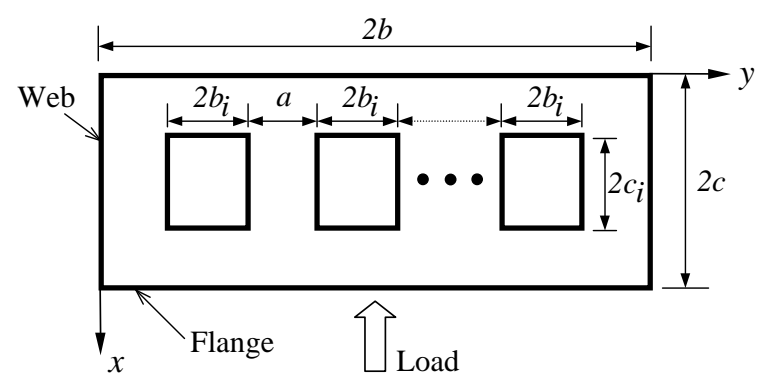

Fig. 6

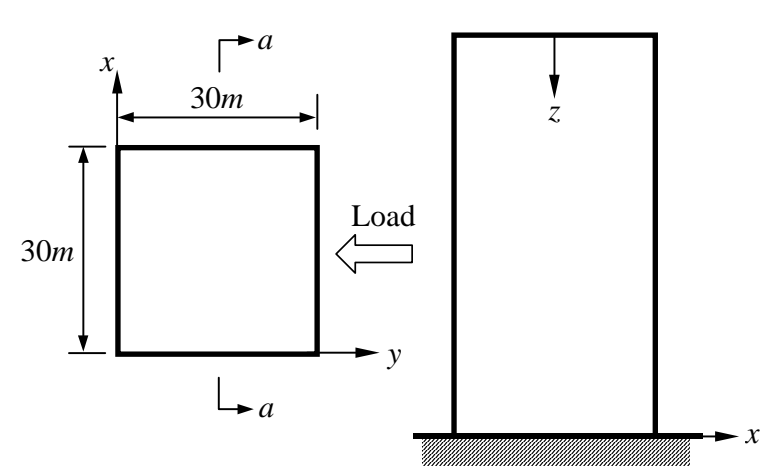

Fig. 7 


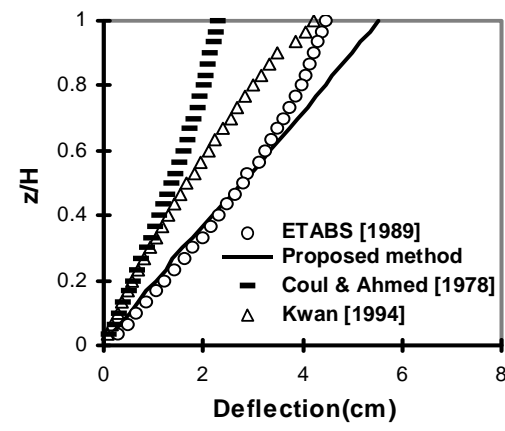

(a) 30-storey

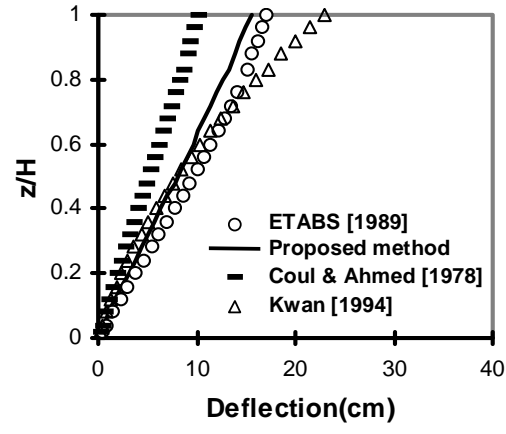

(b) 50-storey

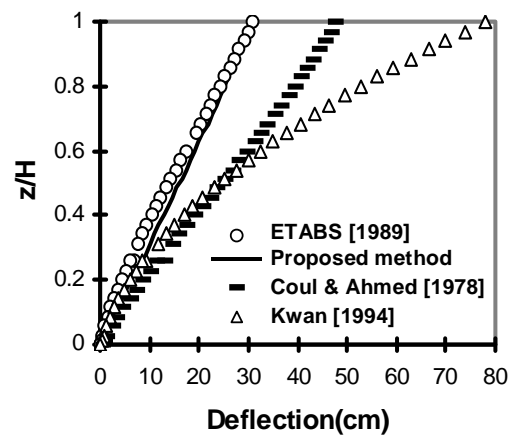

(c) 70-storey

Fig. 8 

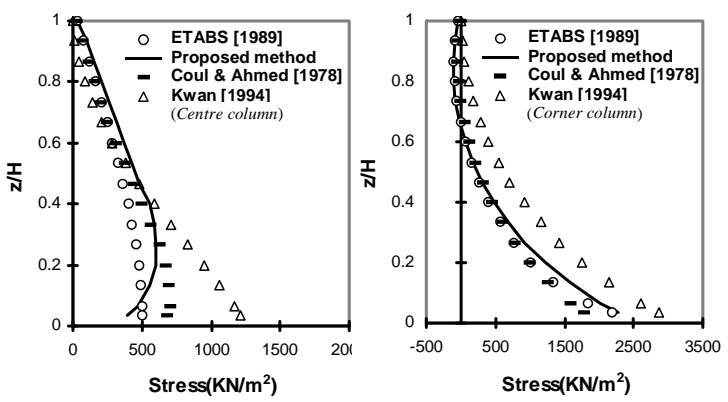

(a) 30-storey
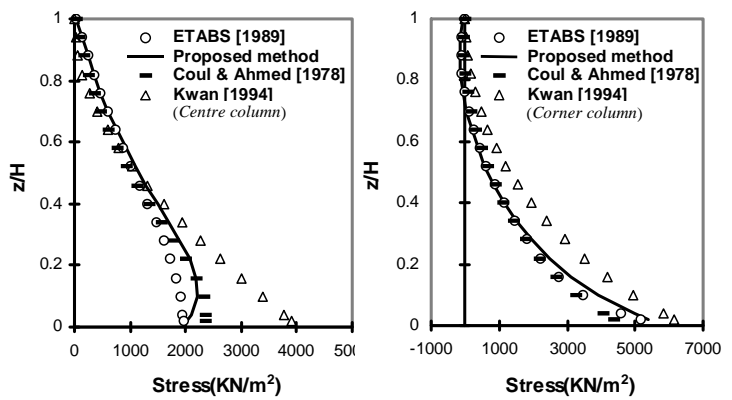

(b) 50-storey
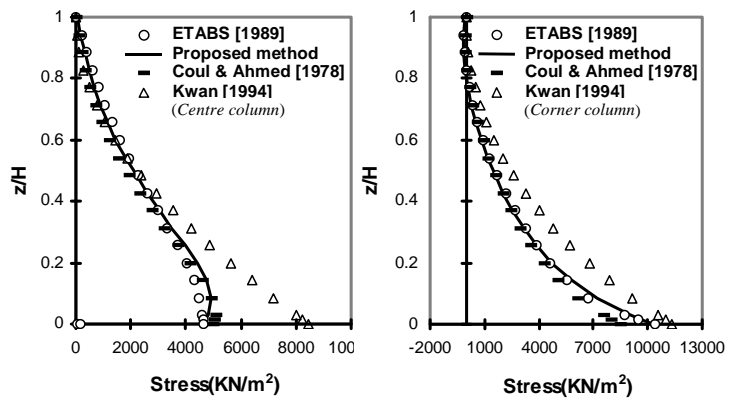

(c) 70-storey

Fig. 9

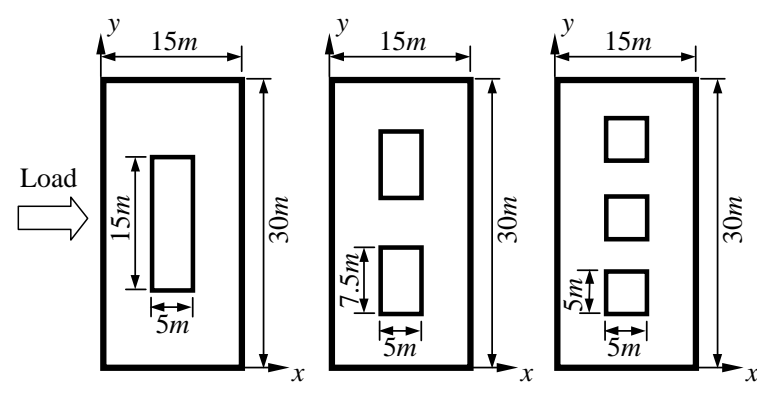

Fig. 10 


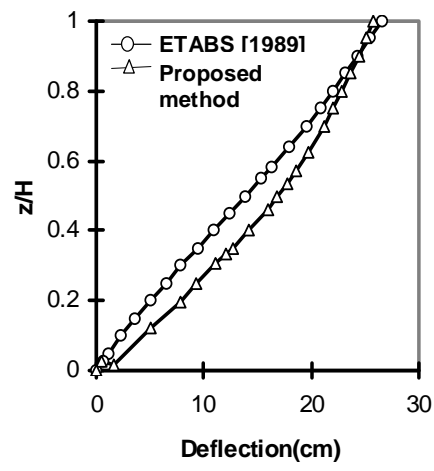

(a) Tube-in-tube structure

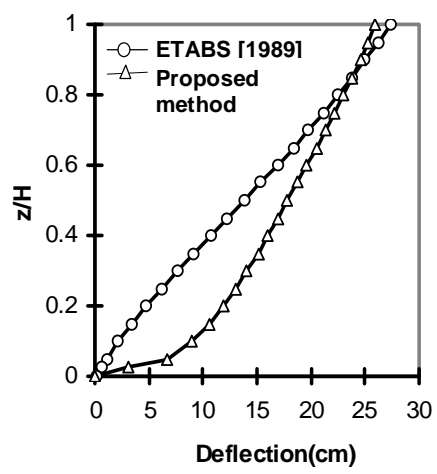

(b) 2 tubes-in-tube structure

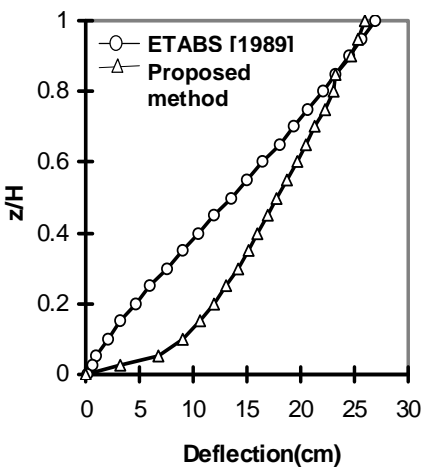

(c) 3 tubes-in-tube structure

Fig. 11 

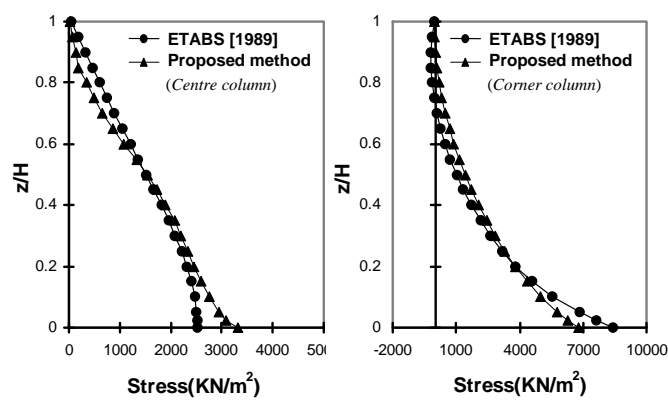

External Tube
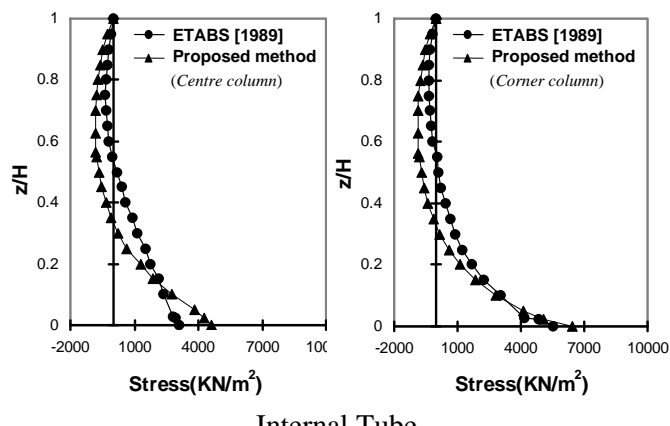

Internal Tube

(a) Tube-in-tube structure
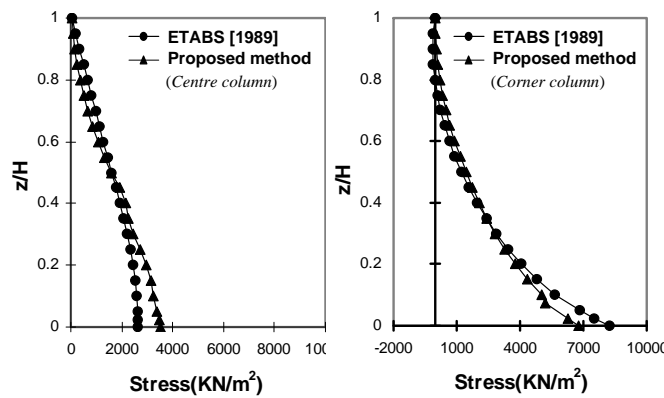

Stress $\left(\mathrm{KN} / \mathrm{m}^{2}\right)$

External Tube
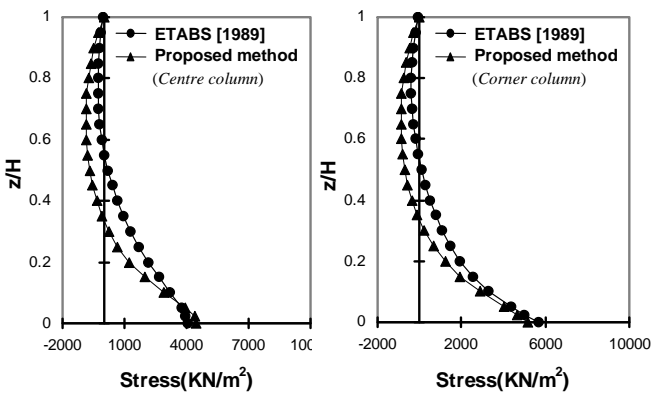

Internal Tube

(b) 2 tubes-in-tube structure

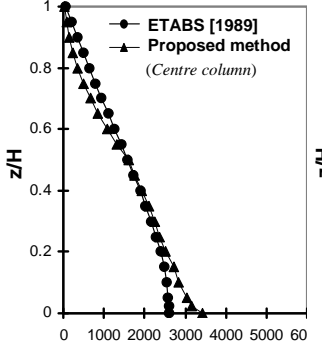

Stress $\left(\mathrm{KN} / \mathrm{m}^{2}\right)$

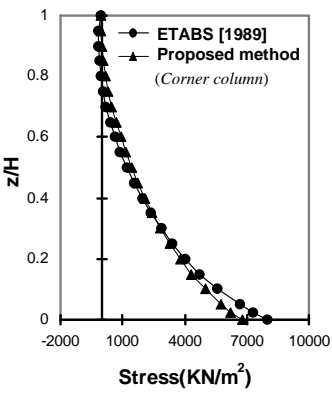

External Tube
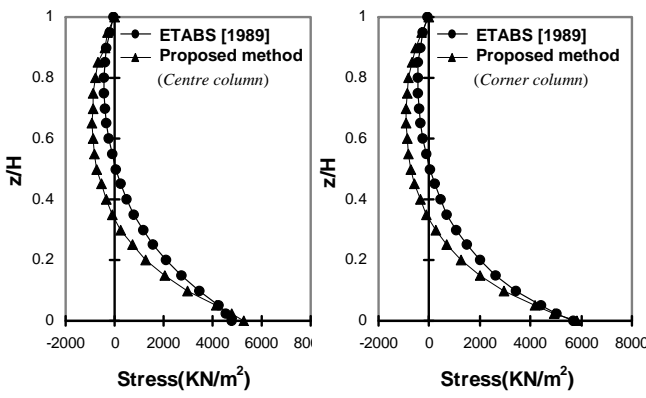

Internal Tube

(c) 3 tubes-in-tube structure

Fig. 12 


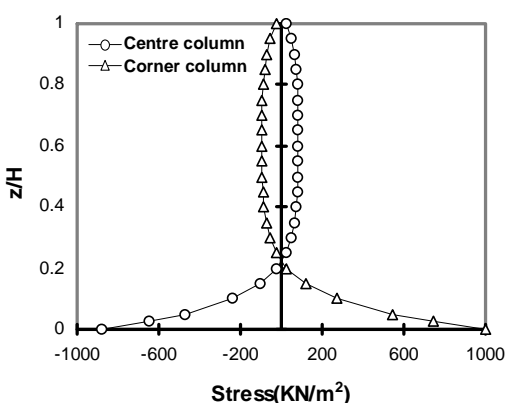

External tube

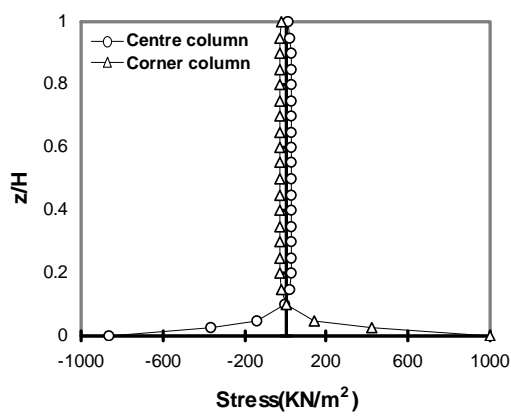

Internal tube

(a) Tube-in-tube structure

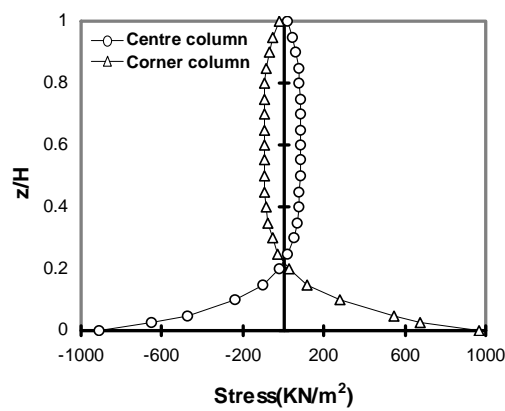

External tube

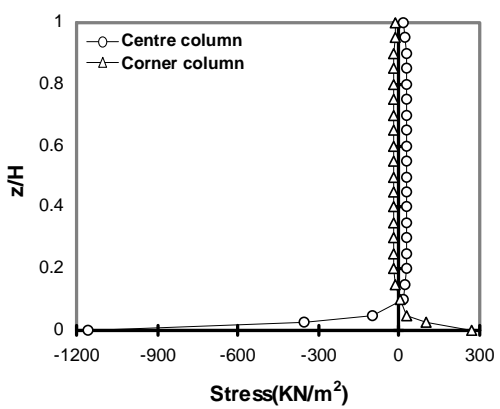

Internal tube

(b) 2 tubes-in-tube structure

Fig. 13

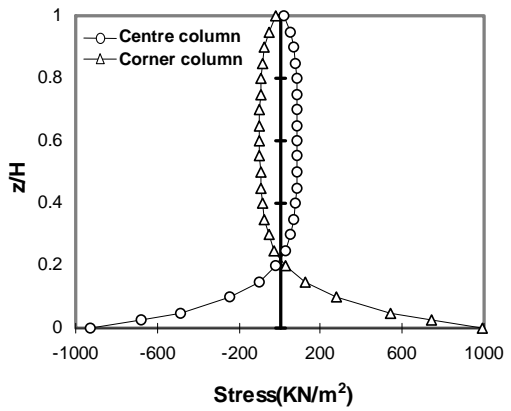

External tube

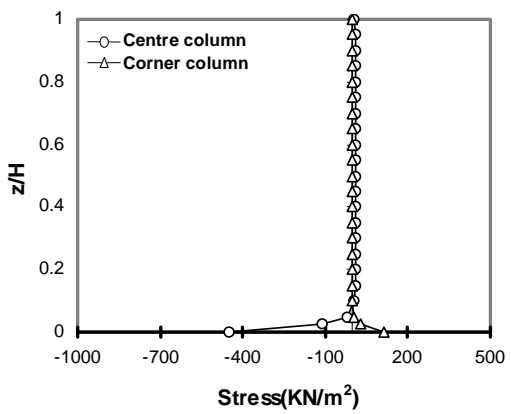

Internal tube

(c) 3 tubes-in-tube structure 